This item was submitted to Loughborough's Research Repository by the author.

Items in Figshare are protected by copyright, with all rights reserved, unless otherwise indicated.

\title{
Nonequilibrium molecular dynamics simulations of nanoconfined fluids at solid-liquid interfaces
}

PLEASE CITE THE PUBLISHED VERSION

http://dx.doi.org/10.1063/1.4986904

PUBLISHER

AIP Publishing

VERSION

NA (Not Applicable or Unknown)

LICENCE

CC BY-NC-ND 4.0

\section{REPOSITORY RECORD}

Morciano, M., M. Fasano, Andreas Nold, C.C. Braga, P. Yatsyshin, David Sibley, Benjamin D. Goddard, E. Chiavazzo, P. Asinari, and Serafim Kalliadasis. 2019. "Nonequilibrium Molecular Dynamics Simulations of Nanoconfined Fluids at Solid-liquid Interfaces". figshare. https://hdl.handle.net/2134/25563. 


\section{Nonequilibrium molecular dynamics simulations of nanoconfined fluids at solid- liquid interfaces}

M. Morciano, M. Fasano, A. Nold, C. Braga, P. Yatsyshin, D. N. Sibley, B. D. Goddard, E. Chiavazzo, P. Asinari, and S. Kalliadasis

Citation: The Journal of Chemical Physics 146, 244507 (2017); doi: 10.1063/1.4986904

View online: https://doi.org/10.1063/1.4986904

View Table of Contents: http://aip.scitation.org/toc/jcp/146/24

Published by the American Institute of Physics

\section{Articles you may be interested in}

Water flow in carbon nanotubes: The effect of tube flexibility and thermostat

The Journal of Chemical Physics 146, 234701 (2017); 10.1063/1.4985252

Wetting at the nanoscale: A molecular dynamics study

The Journal of Chemical Physics 146, 114704 (2017); 10.1063/1.4978497

Analysis of shear viscosity and viscoelastic relaxation of liquid methanol based on molecular dynamics simulation and mode-coupling theory

The Journal of Chemical Physics 146, 244506 (2017); 10.1063/1.4990408

The entropy of a complex molecule

The Journal of Chemical Physics 146, 224106 (2017); 10.1063/1.4984965

Liquid-liquid phase transition in an ionic model of silica

The Journal of Chemical Physics 146, 234503 (2017); 10.1063/1.4984335

Contact angles from Young's equation in molecular dynamics simulations

The Journal of Chemical Physics 147, 084708 (2017); 10.1063/1.4994088

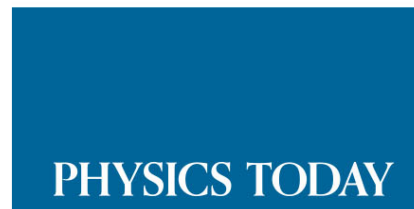




\title{
Nonequilibrium molecular dynamics simulations of nanoconfined fluids at solid-liquid interfaces
}

\author{
M. Morciano, ${ }^{1}$ M. Fasano, ${ }^{1}$ A. Nold,${ }^{2}$ C. Braga,${ }^{2}$ P. Yatsyshin, ${ }^{2}$ D. N. Sibley,${ }^{3}$ \\ B. D. Goddard, ${ }^{4}$ E. Chiavazzo, ${ }^{1}$ P. Asinari, ${ }^{1, a)}$ and S. Kalliadasis $\left.{ }^{2, b}\right)$ \\ ${ }^{1}$ Energy Department, Politecnico di Torino, Corso Duca degli Abruzzi 24, Torino 10129, Italy \\ ${ }^{2}$ Department of Chemical Engineering, Imperial College London, London SW7 2AZ, United Kingdom \\ ${ }^{3}$ Department of Mathematical Sciences, Loughborough University, Loughborough LE11 3TU, United Kingdom \\ ${ }^{4}$ The School of Mathematics and Maxwell Institute for Mathematical Sciences, The University of Edinburgh, \\ Edinburgh EH9 3JZ, United Kingdom
}

(Received 12 February 2017; accepted 7 June 2017; published online 30 June 2017)

\begin{abstract}
We investigate the hydrodynamic properties of a Lennard-Jones fluid confined to a nanochannel using molecular dynamics simulations. For channels of different widths and hydrophilic-hydrophobic surface wetting properties, profiles of the fluid density, stress, and viscosity across the channel are obtained and analysed. In particular, we propose a linear relationship between the density and viscosity in confined and strongly inhomogeneous nanofluidic flows. The range of validity of this relationship is explored in the context of coarse grained models such as dynamic density functional-theory. Published by AIP Publishing. [http://dx.doi.org/10.1063/1.4986904]
\end{abstract}

\section{INTRODUCTION}

The development of novel nanotechnological applications and advanced materials with properties defined by transport phenomena at the nanoscale demands an improved understanding of rheology and tribology at the molecular level. For example, quantifying the rheological properties of fluids confined within nanoscale cavities may enable more efficient design of lab-on-a-chip applications. Also, viscosity regulates the pumping power needed to operate fluids in energy and lubricating applications ${ }^{1-3}$ and affects drug release rates in biomedical applications. ${ }^{4,5}$ Molecular modelling of fluid flow in the vicinity of a solid substrate is also of fundamental interest, as it involves a wide spectrum of non-trivial physical phenomena across scales, such as hard-sphere repulsion, slip, viscous forces, and disjoining pressure effects. ${ }^{3,6-13}$ As such it can serve as a paradigm for physical systems exhibiting multiscale dynamics.

All the above physical and technological settings involve fluid flow in contact with solid surfaces. The associated boundary condition has deserved particular attention over many years, going back to the work of Navier. ${ }^{14}$ However, at the nanoscale, classical continuum hydrodynamic models, such as the Navier-Stokes (NS) equations, are not able to accurately describe fluid flow and properties. ${ }^{15-18}$ In fact, they break down when the atomic nature of matter results in state variables (e.g., density) changing significantly on a scale that is commensurate with the molecular mean free path, which is certainly the case in the vicinity of a solid boundary. One consequence is that the classical no-slip boundary condition for fluid flow past a solid surface ${ }^{18,19}$ is not valid for nanoscale flows, e.g., flows in nanochannels. ${ }^{15-18}$ In particular, at the nanoscale,

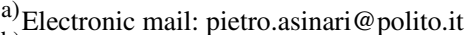

b) Electronic mail: s.kalliadasis@imperial.ac.uk
}

transport properties depend on highly inhomogeneous fluid properties, leading to a behaviour which is inconsistent with NS dynamics. ${ }^{20}$

Therefore, the no-slip condition needs to be modified appropriately. Unfortunately, experimental analysis at the nanoscale is particularly difficult and plagued by measurement errors. Molecular dynamics (MD) provides closure relationships to hydrodynamics from a more fundamental physical level. For example, this approach is able to evaluate the slip velocities which cannot be done with standard hydrodynamics calculations.

In their groundbreaking study, Thompson and Troian investigated slip between a Lennard-Jones (LJ) fluid and solid walls, showing that the no-slip condition was justified at sufficiently low shear rates but failed at higher shear rates, at which the slip length diverges as a critical shear rate is approached. ${ }^{18,21-23}$

As far as the bulk quantities for self-diffusivity and viscosity of fluids are concerned, these have been studied extensively using MD simulations. For instance, Rowley and co-workers ${ }^{24}$ reported MD computations of the self-diffusion coefficient and the viscosity in agreement with argon experimental data. Later, Meier and co-workers ${ }^{25}$ calculated the kinetic and potential viscosity contributions for a broad range of temperatures and densities. It was also shown that results for LJ fluids may be extrapolated to real fluids using a corresponding states scheme. ${ }^{26}$

Studies for the viscosity in confined geometries were undertaken by Hartkamp and co-workers ${ }^{27,28}$ for the case of a planar Poiseuille flow, uncovering a highly oscillatory behaviour close to the wall, which depends on the applied body force. Other studies focus on identifying the nonNewtonian part of the stress tensor of a sheared fluid. ${ }^{29}$ Our primary aim here is to explore the possibility of universal relations for transport properties, such as the shear viscosity 
close to solid substrates in confined geometries, by considering a prototype, the (well-studied) planar Couette flow in a nanochannel.

Ultimately, finding universal relationships between the stress tensor, the density distribution, and the velocity field would allow us to formulate a closure for nanoscale continuum models. For instance, recent works based on the statistical mechanics of classical fluids, namely, density functional theory (DFT), have shown that fluid layering gives a rich structure near contact lines. ${ }^{10,30}$ This structure is expected to also impact dynamic situations, which may be modelled using continuum dynamic DFT (DDFT) for colloidal fluids. Bridging the gap between DDFT and NS requires relaxation of the main assumptions inherent to colloidal fluids leading to an NS-like equation. ${ }^{31,32}$

However, in this equation a number of closures remain unspecified, such as the relation between viscosity and density, which is crucial where we have clustering of molecules, i.e., close to boundaries such as walls and interfaces. The use of empirical expressions between the viscosity and density leaves open questions as to how accurately DDFT models and the NS-like equation obtained in Refs. 31 and 32 can capture the correct physics in dynamic settings. Part of the aim of the present work aims to test such empirical relations for nanoconfined fluids.

For this purpose, MD simulations are performed for a model system, a LJ fluid confined between LJ walls. Using a variety of channel width and shear rates, we scrutinise the slip length, the spatially dependent number density, and the stress tensor of the LJ fluid in contact with the sheared wall for various shear rates and channel widths.

Our computations reveal a strong anisotropic behaviour of the mass transport properties of the fluid along the channel section due to the solid-liquid non-bonded interactions in the layers close to the LJ walls. In particular, we probe the quality of a linear relation between viscosity and density, applied throughout the channel, and exclusively based on parameters extracted from the bulk fluid. We also test the impact of a refined relationship, by including a linear dependency on the density gradient. Interestingly, apart from the first fluid layer close to the wall, the simplest law solely based on bulk values already provides a good agreement with our MD simulations. Including a linear dependency on the density gradients in a confinement-independent manner further improves the quality of the prediction.

Whilst these observations may not be used to replace effective slip conditions, which heavily depend on the fluid behaviour in the first fluid layer, they may serve as a guide for the description of fluid flows with highly inhomogeneous density distributions. For instance, the laws presented in this work may be used in coarse-grained nanoscale continuum DDFT and NS-like models, in order to study moving contact lines or fluids in nanoconfinement. In these highly inhomogeneous situations, capturing the viscosity accurately away from the wall is essential in order to predict macroscopic quantities such as the contact line friction.

In Sec. II, we describe our MD methodology. Our results are discussed and analysed in Sec. III, and overall discussions and conclusions are offered in Sec. IV.

\section{METHODS}

One of the fundamental goals of liquid state theory is to understand and predict the behaviour of a fluid based on the properties of its constituent molecules. ${ }^{33}$ For this purpose, MD stands out as an attractive simulation method that explicitly takes into account the molecular nature of liquids and is therefore able to calculate the fluid transport properties at the molecular scale.

The fluid is modelled as a set of monoatomic particles interacting with both fluid and wall particles via a 12-6 LJ potential $U$,

$$
U_{i j}(r)=4 \varepsilon_{i j}\left[\left(\frac{\sigma_{i j}}{r}\right)^{12}-\left(\frac{\sigma_{i j}}{r}\right)^{6}\right],
$$

where $r$ is the atom-atom distance, $\sigma_{i j}$ is the range parameter, namely, the distance at which the inter-particle potential is zero, $\varepsilon_{i j}$ is the depth of the potential well, and $\{i, j\}$ take values as either $w$ or $f$ for wall or fluid particles. The mixed parameters are chosen according to the Lorentz-Berthelot combination rules ${ }^{34}$

$$
\sigma_{i j}=\frac{\sigma_{i}+\sigma_{j}}{2}, \quad \varepsilon_{i j}=\sqrt{\varepsilon_{i} \varepsilon_{j}} .
$$

In what follows, we drop the subscript $f . m$ is the mass of a fluid particle and the characteristic time is $\tau=\sqrt{\sigma^{2} m / \varepsilon}$.

Our MD simulations are performed using the Large-Scale Atomic/Molecular Massively Parallel Simulator (LAMMPS) ${ }^{35}$ Specifically, we simulate an NVT ensemble by integrating Newton's equations of motion using a version of the Verlet algorithm ${ }^{34,35}$ with a time step of $d t=0.005 \tau .^{18,21}$ The initial velocities of the fluid particles are sampled from a Maxwell-Boltzmann distribution, with a variance corresponding to the temperature $T$.

The fluid is confined between rigid walls. A Cartesian coordinate system $(x, y, z)$ is introduced with an origin such that $y=y_{0}$ is on the lower wall, where $y_{0}$ is the position of the wall layer contacting the bottom of the fluid (here $3.75 \sigma$ ), and with $x$ the streamwise coordinate, $y$ the outward-pointing coordinate normal to the lower wall, and $z$ the spanwise coordinate, see Fig. 1. The fluid flow is induced by moving the top wall, thus generating a planar Couette flow. The accessible Couette cell (excluding the walls) measures $[21.368 \sigma] \times[h] \times[12.792 \sigma]$, along the $x, y$, and $z$ axes, respectively. Values for $h$ vary from

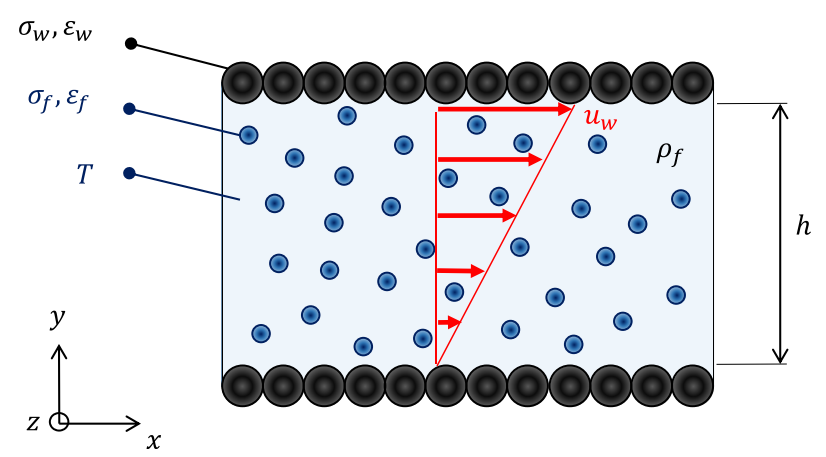

FIG. 1. Sketch of the atomistic view of a Couette flow of a LJ fluid at temperature $T$ through a channel of width $h$. The top wall is sheared with velocity $u_{w}$. The velocity profile of the flow is shown by the red arrows. The fluid and wall LJ parameters are $\varepsilon_{f}, \sigma_{f}$ and $\varepsilon_{w}, \sigma_{w}$, respectively. 
$6.43 \sigma$ to $21.44 \sigma$, representing different degrees of nanoconfinement. Periodic boundary conditions are applied along the $x$ and $z$ directions. ${ }^{35}$ The wall velocities are chosen to be large enough to attain a high signal-to-noise ratio while avoiding the shear thinning regime of the fluid.

Each fluid atom is partially (i.e., only in the z-direction to avoid biasing the flow) and weakly coupled to a heat bath by a Langevin thermostat. ${ }^{18,35-38}$ Thus, a constant temperature is maintained along the channel (see Appendix A). With the Langevin thermostat, the total force acting on the $k$ th atom is

$$
\mathbf{F}_{\mathrm{tot}, k}=\mathbf{F}_{c, k}+\mathbf{F}_{r, k}+\mathbf{F}_{t, k},
$$

where $\mathbf{F}_{c, k}$ is the $\mathrm{LJ}$ force

$$
\mathbf{F}_{c, k}=-\sum_{\ell \neq k} \boldsymbol{\nabla} U_{k \ell}
$$

$\mathbf{F}_{r, k}$ represents a frictional drag term proportional to the particle mass $m_{k}$ and velocity $\mathbf{v}_{k}$ as

$$
\mathbf{F}_{r, k}=-m_{k} \Gamma\left(\mathbf{v}_{k} \cdot \mathbf{e}_{z}\right) \mathbf{e}_{z},
$$

where $\Gamma$ is the friction constant controlling the rate of the heat exchange with the reservoir. Finally, $\mathbf{F}_{t, k}$ is a Gaussian distributed random force with zero mean and non-zero variance

$$
\mathbf{F}_{t, k}=\sqrt{\frac{2 k_{B} T m_{k} \Gamma}{\Delta t}}\left(\hat{\mathbf{k}}_{k} \cdot \mathbf{e}_{z}\right) \mathbf{e}_{z},
$$

where $T$ is the fluid temperature, $\Delta t$ is the time step, $k_{B}$ is the Boltzmann constant, and $\hat{\mathbf{k}}_{k}$ is a random fluctuating vector with a magnitude given by the Gaussian distribution with unit variance and mean zero. In detail, the equation of motion in the $z$-direction for the $k$ th fluid particle is thus given by

$$
m_{k} \ddot{z}_{k}=-\sum_{\ell \neq k} \frac{\partial U_{k \ell}}{\partial z_{k}}-m_{k} \Gamma \dot{z_{k}}+\sqrt{\frac{2 k_{B} T m_{k} \Gamma}{\Delta t}} \hat{k}_{k},
$$

where $\ddot{z}_{k}$ is the acceleration of the $k$ th fluid particle along the $z$-direction. The last two terms disappear in the directions which define the shear plane. ${ }^{39}$ In these directions, Newton's equations are solved.

By applying the Langevin thermostat, every particle is coupled to a viscous background and a stochastic heat bath. ${ }^{36,40-43}$ The friction coefficient, which regulates the heat flux from the simulation domain, should be large enough to dissipate all the generated heat without causing significant changes in temperature but small enough not to affect the particle trajectories. ${ }^{23,36,44,45}$ Consequently, in this work, $\Gamma=1.0 \tau^{-1}$ in line with Refs. $18,21,40$, and 46-49.

The alternative thermostating scheme, where only the wall atoms have the thermostat applied, is unfortunately not efficient for the studied systems. In particular, the work done by the applied shear raises the temperature of the fluid to values that are too large to be efficiently removed by heat conduction with the wall particles. This behaviour can be observed in the work of Yong and Zhang ${ }^{36}$ where the fluid temperature in the middle of the channel becomes significantly larger than that near the walls, thus raising the question of the validity of the corresponding thermostat choice.

The practical implementation of the described procedure applies a Langevin thermostat locally for each fluid slab element, ensuring that local thermodynamic equilibrium is valid across the system. ${ }^{36}$ The appropriateness of the described method and experimental setup is ensured by checking that the bulk viscosity is in agreement with the value reported in the literature ${ }^{25,26,50}$ and obtained using different approaches including equilibrium MD simulations. Also, here the simulated fluid number density $\rho=0.82 \sigma^{-3}$ is exactly that of the coexisting bulk liquid at temperature $T=1.1 \varepsilon / k_{B}{ }^{18}$

The mass of the wall atoms has been chosen as $m_{w}$ $=2.0 \mathrm{~m}$. Both the top and the bottom walls are effectively impenetrable surfaces, and the wall atoms are rigidly fixed in the lattice sites of a simple cubic cell, ${ }^{36}$ with a lattice site length of $1.072 \sigma$. The walls consist of 4 layers of atoms (i.e., 960 atoms per wall). Thus, by considering the different degrees of fluid nanoconfinement, the number of atoms in the simulation domain ranges from 3360 to about 10100.

The LJ parameters are set to $\sigma_{w}=1.0 \sigma$; to model different wall-fluid interactions, we choose $\varepsilon_{w}=1.0 \varepsilon$ for a hydrophilic configuration $\left(\varepsilon_{w} \geq \varepsilon\right)$ and $\varepsilon_{w}=0.2 \varepsilon$ for a hydrophobic configuration $\left(\varepsilon_{w}<\varepsilon\right)$. The cutoff radius of the LJ potential is set to $r_{c}=2.5 \sigma$, a commonly used value balancing computational speed with accuracy. ${ }^{34}$

The simulation protocol consists of three steps. First, the confined fluid is equilibrated within $2 \times 10^{6} \tau$ time steps. Second, a velocity gradient is applied by moving the top wall for $2 \times 10^{6} \tau$ time steps. Third, once the velocity profile of the confined fluid is in a steady state, simulations are continued up to $20 \times 10^{6} \tau$, to ensure statistical relevance of the measured fluid properties. Fluid properties are both time- and space-averaged along steady-state trajectories. The spatially varying quantities (e.g., fluid density and average velocity) are computed by subdividing space into bins of widths that provide a satisfactory compromise between statistical noise and resolution.

The fluid density is computed using 750 and 500 slabs for $h=21.44 \sigma$ and $h=6.43 \sigma$, respectively. Due to higher levels of noise, the velocity profiles for the hydrophilic and the hydrophobic case are computed using bin widths of $\Delta h=0.05 h$ and $\Delta h=0.025 h$, respectively. All quantities are given as bin and time averages along the simulated domain and trajectories, respectively. For calculations where a higher resolution is required, standard linear interpolation is used.

\section{RESULTS}

To obtain a qualitative picture of the mass transport properties of a LJ fluid, we perform MD simulations in channels of width $h=6.43 \sigma$ and $21.44 \sigma$, allowing us to investigate the influence of confinement on the flow. By changing the strength of the LJ fluid-substrate interactions $\varepsilon_{w}$, we simulate hydrophilic $\left(\varepsilon_{w}=1.0 \varepsilon\right)$ and hydrophobic $\left(\varepsilon_{w}=0.2 \varepsilon\right)$ substrates. Finally, we consider different strain rates by changing the velocity of the sheared wall. From these simulations, we obtain a comprehensive picture of the effects of confinement and fluid-wall attraction by analysing the density structure, velocity profiles, slip (see Appendix C), fluid 
stresses, and viscosities. This finally allows us to validate a linear constitutive relationship between the density and the viscosity, which may be then used in coarse-grained models such as the NS-like equation obtained in Refs. 31 and 32.

\section{A. Equilibrium density profiles}

We begin by computing the equilibrium fluid density distributions across the nanochannel. Several representative density profiles corresponding to hydrophilic and hydrophobic walls and two different channel widths are depicted in Fig. 2.

The fluid density profiles exhibit an oscillatory behaviour near the substrate walls, which for the chosen value of fluid temperature $\left(T=1.1 \varepsilon / k_{B}\right)$ persists up to distances of 4-6 particle diameters from the walls. ${ }^{51-56}$ The stronger the attraction of the hydrophilic substrate is, the more pronounced the oscillations in the near-wall fluid structure become. In particular, in the wider channel, the height of the first density peak near the hydrophilic wall is about $2.75 \sigma^{-3}$, whereas the corresponding value of the first peak near the hydrophobic wall is about $1.6 \sigma^{-3}$. In all the profiles presented, the density peaks are set approximately one hard core diameter apart. This oscillatory near-wall fluid structure is a manifestation of the correlated short-range intermolecular repulsions, which play a key role during layering and freezing transitions in adsorbed fluids. ${ }^{57}$

As it can be seen in the case of the wider channel in Fig. 2(b), the amplitude of the density oscillations rapidly decays while approaching the bulk volume of the fluid. ${ }^{18,23,51,58}$ It is noteworthy that the values of the density at the near-constant plateaus in the center of the channel are slightly, but noticeably, different for the hydrophilic and hydrophobic cases. This is a manifestation of the Kelvin shift of the bulk coexistence curve, which occurs due to the spatial confinement of the fluid, ${ }^{59}$ reflecting the higher degree of effective confinement in the hydrophobic case due to the repulsive wall layer. In fact, the underlying physics can give rise to rich phase equilibria and wetting transitions for capillaries, ${ }^{60}$

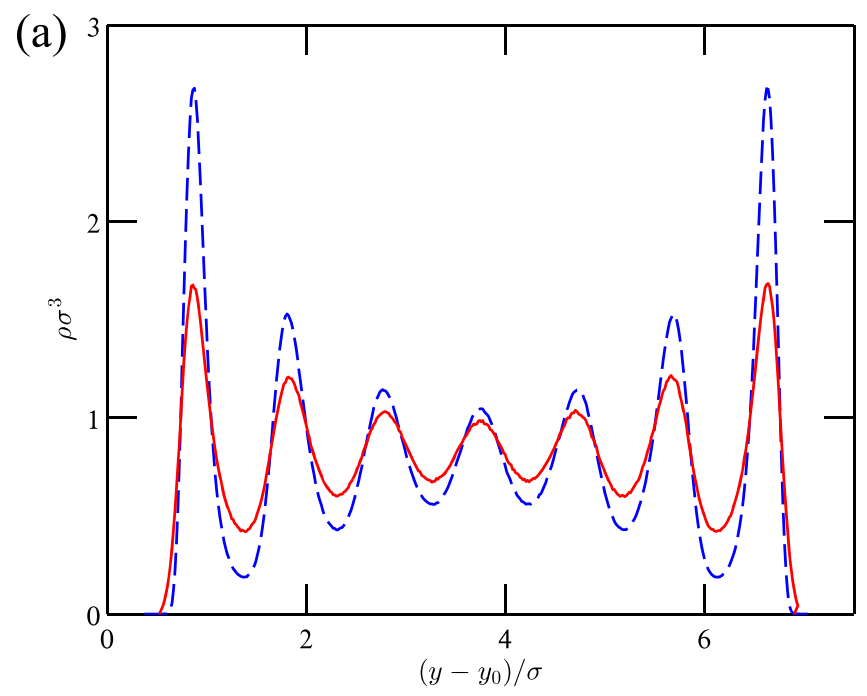

grooves, ${ }^{61,62}$ and more general geometries such as steps and wedges. $^{63}$

We note that in the more confined case presented in Fig. 2(a), the channel is too narrow for the fluid density to be able to reach a near-constant plateau. It is also worth pointing out that the simplified model of the fluid used in this work (i.e., a LJ fluid) tends to exhibit greater structuring close to the wall in comparison to fluids involving electrostatic interactions and multiple points. However, in the present study, the main interest is the breakdown of the constitutive laws for highly structured fluid systems; therefore, a limiting case is useful to understand and quantify molecular effects and to investigate the fluctuations which take place in the fluid. More complex models (e.g., SPC/E or TIP4P in the case of water) should then be exploited to extend current results to fluids with properties closer to experimental ones. 7

\section{B. Velocity profiles}

The velocity profiles are computed at different shear rates ranging from $\gamma=0.063 \tau^{-1}$ to $0.280 \tau^{-1}$. Wall velocities are chosen to be sufficiently large to obtain high signal-to-noise ratios, while at the same time care is taken to avoid the shear thinning regime. In this case, the fluid atoms cannot respond fast enough to the deformation due to shear and the confined fluid forms ordered structures, which promotes the sliding motion between different layers and leads to reduced viscosity. $^{51}$

As described by Yong and Zhang, ${ }^{36}$ in the case where the Langevin thermostat is applied to the fluid particles, accurate dynamics with small disturbances to the particle trajectories can be achieved in weakly sheared systems. Thus, here we focus our attention on the dynamics produced at the lowest shear rate. In Fig. 3, we show velocity profiles of the fluid confined in hydrophilic and hydrophobic channels of widths $h=6.43 \sigma$ to $21.44 \sigma$.

For strongly wetting walls (see top plot in Fig. 3), the velocity of the fluid relative to the wall vanishes at the

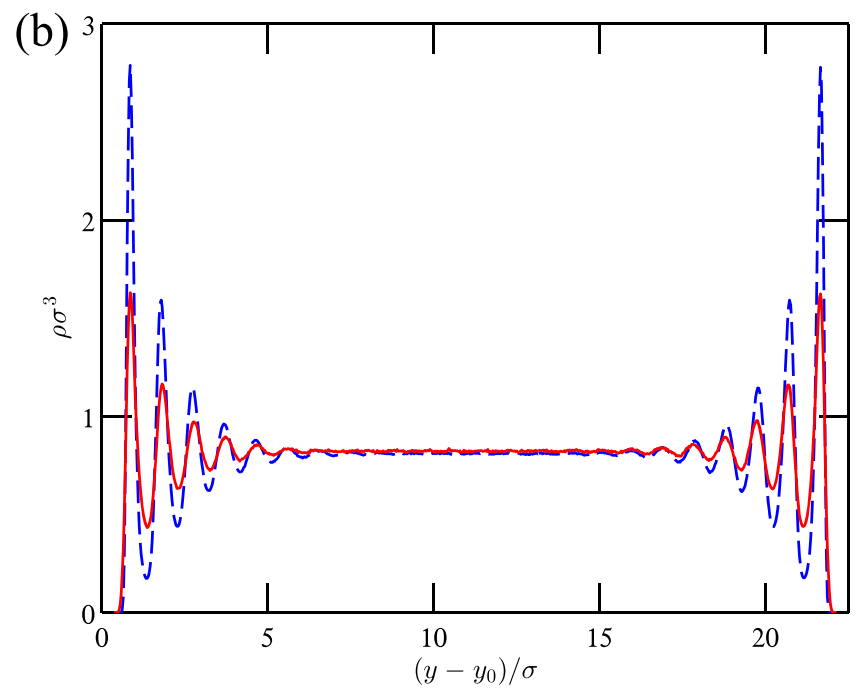

FIG. 2. Fluid density profiles, $\rho$, across the nanochannels of width (a) $h=6.43 \sigma$ and (b) $h=21.44 \sigma$. Blue dashed and red solid curves correspond to the hydrophilic $\left(\varepsilon_{w}=1.0 \varepsilon\right)$ and hydrophobic $\left(\varepsilon_{w}=0.2 \varepsilon\right)$ walls, respectively. 

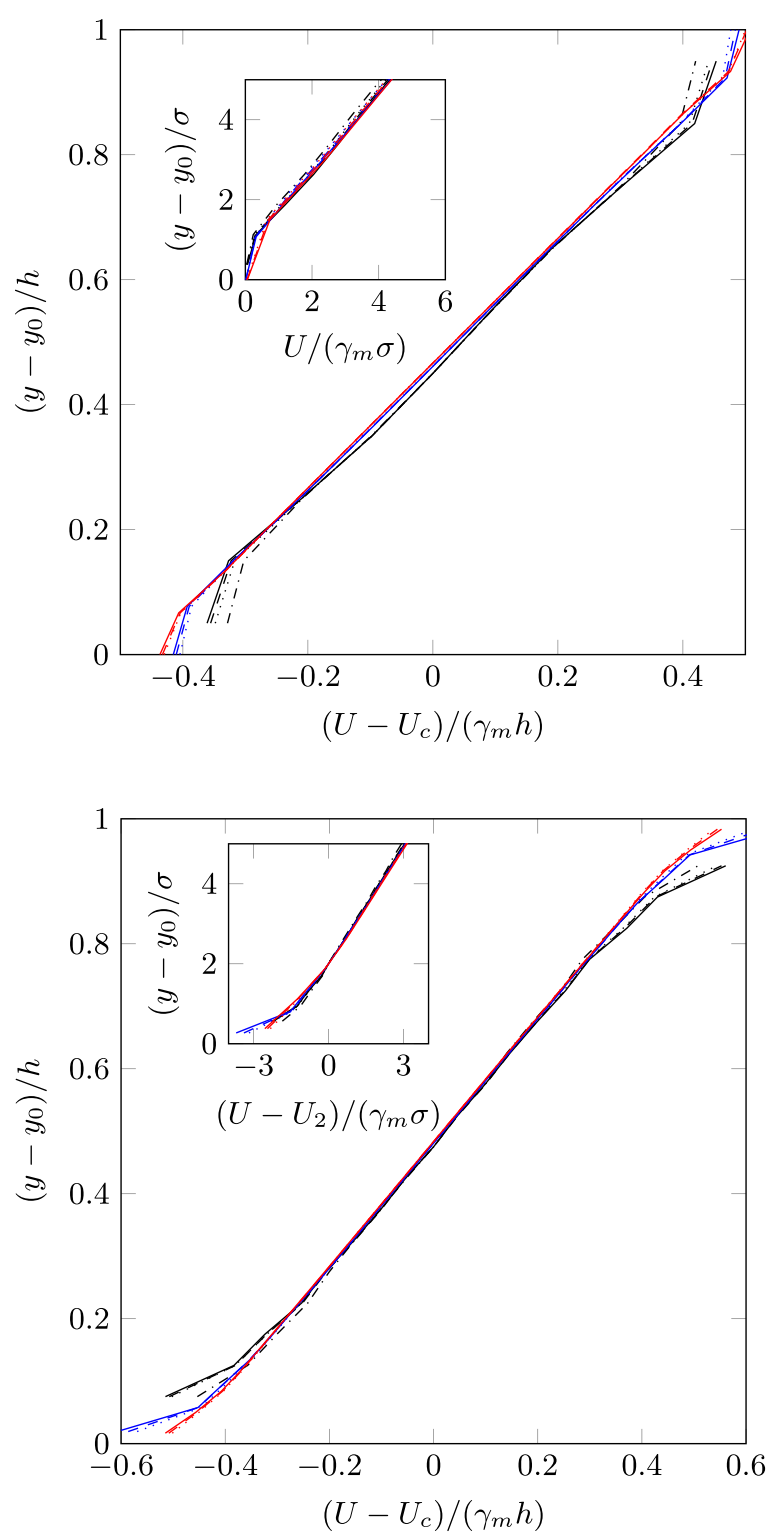

FIG. 3. Velocity profiles for the hydrophilic $\left(\varepsilon_{w}=1.0 \varepsilon\right.$, top) and the hydrophobic $\left(\varepsilon_{w}=0.2 \varepsilon\right.$, bottom) cases. Black, blue, and red lines represent data for channels with height $h=6.43 \sigma, 12.86 \sigma$, and $21.44 \sigma$, respectively. The solid, dashed, dotted, and dashed-dotted lines are results for shear rates $\gamma=0.28 \tau^{-1}, 0.233 \tau^{-1}, 0.186 \tau^{-1}$, and $0.063 \tau^{-1}$, respectively. The shear rates $\gamma_{m}$ used for the nondimensionalisation were measured in the center of the domain. $U_{2}$ is the velocity at a distance of $2 \sigma$ away from the wall, and $U_{c}$ is the velocity at the center of the domain.

solid-fluid interface. This occurs for each strain rate considered and is due to the large wall-fluid interaction potential. ${ }^{23,44}$ The large momentum transfer at the boundary layer even leads to effective negative slip lengths. Therefore, the fluid layers adsorbed at the wall can be viewed as an extended wall layer, which induces increased shearing in the middle of the fluid by reducing the width of the flow region.

On the other hand, for weaker, i.e., hydrophobic walls (see the bottom plot in Fig. 3), the velocity profile exhibits significant slip, ${ }^{22}$ leading to positive slip lengths of the order of a few hard sphere diameters. Note that the velocity profiles collapse for distances farther than $2 \sigma$ away from the wall.

\section{Stress tensor profiles}

We have studied the fluid stress profiles for different flow and confinement conditions with the aim to understand the relation between density, stress tensor, and viscosity across the channel. The stress tensor computations are based on the Irving-Kirkwood method, ${ }^{64}$ which is composed of a kinetic and a configurational contribution. The kinetic contribution is linked to the local translational shear viscosity and corresponds to the momentum transfer associated with the displacement of the particles. The configurational contribution comes from the intermolecular forces between the particles. ${ }^{25,27,65}$ To leading order, the local Irving-Kirkwood expression ${ }^{66}$ for the $x y$ component of the stress tensor is

$$
\begin{aligned}
\tau_{x y, \text { bin }}(y)= & -\frac{1}{V_{\text {bin }}} \sum_{i \in \text { bin }}^{N_{\text {bin }}}\left(\frac{1}{2} \sum_{j \in \text { fluid }}^{N_{\text {fluid }}}\left(r_{i, x}-r_{j, x}\right) F_{i, j, y}\right) \\
& -\frac{1}{V_{\text {bin }}} \sum_{i \in \text { bin }}^{N_{\text {bin }}}\left(m_{i}\left\langle v_{i, y}\left[v_{i, x}-v_{x, \text { bin }}(y)\right]\right\rangle\right),
\end{aligned}
$$

where $r_{i, x}$ and $r_{j, x}$ are the positions of the interacting atoms, $F_{i, j, y}$ is the interatomic force in the $y$-direction, $N_{\text {bin }}$ is the number of fluid atoms in the bin, $N_{\text {fluid }}$ is the total number of fluid atoms, $V_{\text {bin }}$ is the volume of each bin, and $v_{x \text {,bin }}(y)$ is the mean local streaming velocity of each bin. Note that in our sign convention, the forces are applied to the fluid particles.

The diagonal components of the stress tensor are also depicted in Fig. 4. Evidently, the fluid layers close to the wall show an anisotropic behaviour along the $y$ direction, which is primarily induced by the fluid density oscillations, and is therefore an equilibrium effect. ${ }^{27,28,51}$ We note that due to large wall-fluid interactions, $\sigma_{y y}$ is significantly different from $\sigma_{x x}$ and $\sigma_{z z}$, whereas $\sigma_{x x}$ and $\sigma_{z z}$ show a similar behaviour. As a consequence, the normal stress difference (namely, $\sigma_{y y}-\sigma_{x x}$ ) oscillates between negative and positive values approaching the interface region. In the inner region, instead, solid-fluid interactions are negligible, and bulk conditions are recovered. Our results indicate that the surface influence extends up to 5 molecular diameters from each wall.

The off-diagonal components $\tau_{x z}$ and $\tau_{y z}$ of the stress tensor are depicted in Fig. 4. The mean values of both $\tau_{x z}$ and $\tau_{y z}$ vanish, as expected, since the fluid is only perturbed along the $x$ direction. Due to the confinement in the $y$ direction, an oscillating profile for $\tau_{x y}$ is found, and it is these oscillatory shear-stress profiles which cause complexity in the viscosity of the fluid and which will be explored in detail below.

\section{Viscosity}

In the MD simulations presented here, we have obtained smooth profiles for the velocity, density, and stress, and we therefore explore the possibility to extend the concept of viscosity to nanoscopic flows. We do so by using a linear constitutive Newtonian equation, ${ }^{67}$ an approximation which is expected to be informative to coarse-grained DDFT and NS-like models used to approximate similar confined flows, as they include Newtonian viscosity terms directly, even where density profiles can be strongly inhomogeneous.

We note, however, that in our MD computations this constitutive linear relation between shear stress and strain 

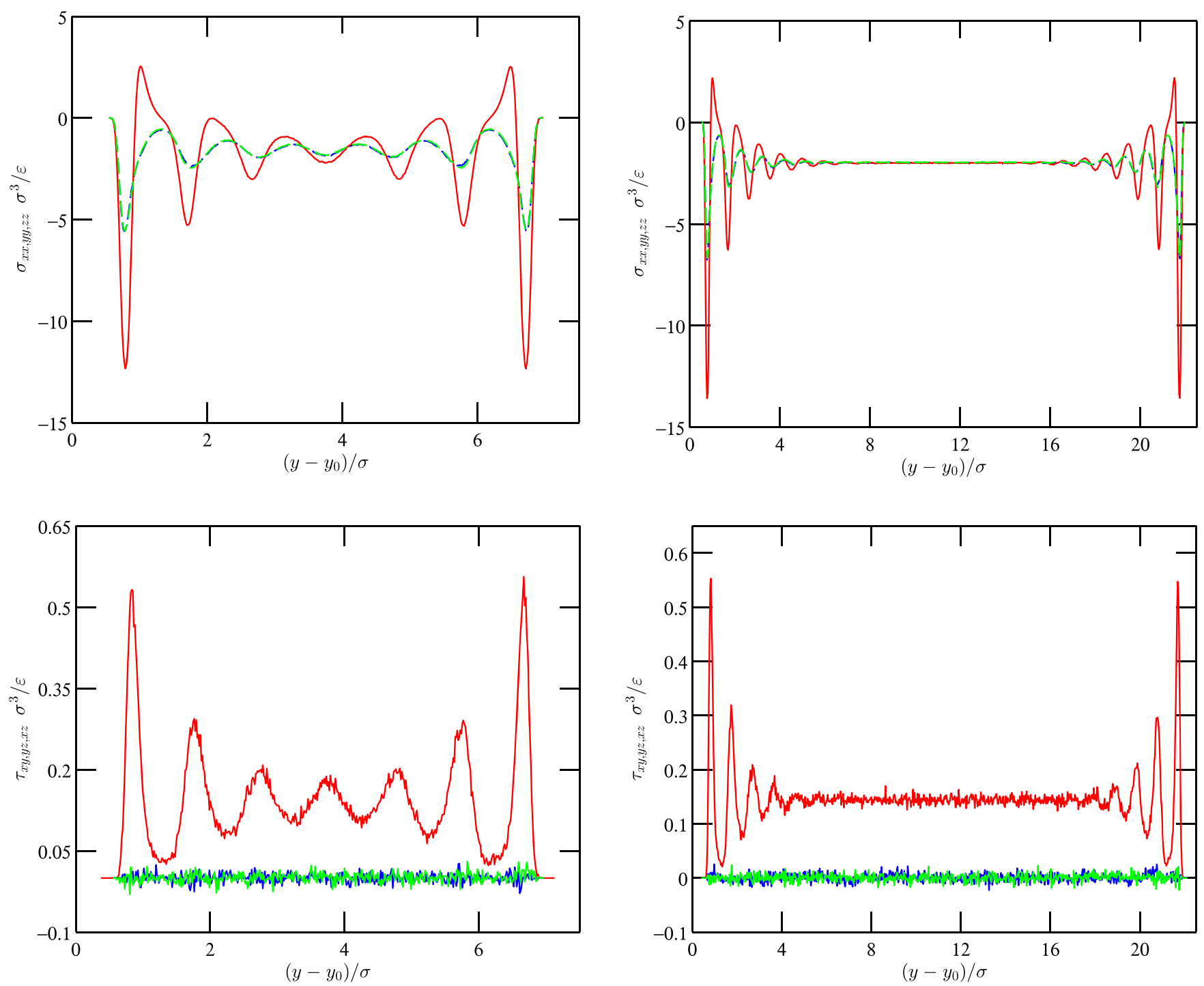

FIG. 4. Top row: normal-virial stress profiles- $\sigma_{x x}$ (dashed green line); $\sigma_{y y}$ (solid red line); $\sigma_{z z}$ (dashed blue line). Bottom row: shear-virial stress profiles- $\tau_{x y}$ (solid red line); $\tau_{x z}$ (dashed green line); $\tau_{y z}$ (dashed blue line). Data are given for hydrophilic substrates $\left(\varepsilon_{w}=\varepsilon\right)$ and shear rate $\gamma=0.063 \tau^{-1}$ for channel widths $h=6.43 \sigma$ and $21.44 \sigma$, shown in the left and right columns, respectively.

rate is likely to break down near the wall where the fluid is strongly inhomogeneous. In particular, at the interface region, the scalar viscosity value must be substituted by a tensorial non-constant quantity. ${ }^{27}$ We stress therefore that whilst the results presented here are adequate for a sheared constrained LJ fluid, they cannot be readily generalised to more complex flow profiles.

Once the stress tensor and velocity profiles are obtained along the channel, it is possible to compute the viscosity profile $\eta(y)$ by exploiting the linear Newtonian relation between the off-diagonal stress tensor and the velocity gradient, namely,

$$
\tau_{x y}=-\eta \frac{\partial v_{x}}{\partial y} .
$$

Figure 5 shows the effect of nanoconfinement on the viscosity $\eta$. In the central region of the nanochannel, the viscosity is roughly constant $\left(\simeq 2.15 \varepsilon \tau / \sigma^{3}\right)$ and in good agreement with the typical bulk values reported in the literature. ${ }^{25,26,50}$ The absence of slip at the solid-fluid interface leads to a viscosity increase with respect to the bulk value within a thin surface layer. ${ }^{28,65}$ For hydrophilic surfaces, the first fluid layer is therefore special in that it is extremely stiff and cannot easily be sheared, in agreement with the no-slip assumption. Moreover, the kinetic contribution is negligible with respect to the configurational one. ${ }^{25,44}$

When the fluid is confined by hydrophobic surfaces, the local viscosity near the wall is lower than in hydrophilic configurations (see the bottom row of Fig. 5), with the near-wall values closer to the bulk ones. The hydrophobic walls cause a greater fluid mobility than the hydrophilic ones, and it is possible to observe the formation of a thin fluid layer close to the surface with strongly reduced viscosity. Because of the reduced viscosity in the proximity of the hydrophobic surfaces, the fluid flow is enhanced within this slip region.

\section{E. Density and viscosity: Hydrophilic substrates}

In Fig. 6, the local relationship between normalised density and normalised viscosity is depicted for different channel widths for hydrophilic substrates. We observe that in the wall 

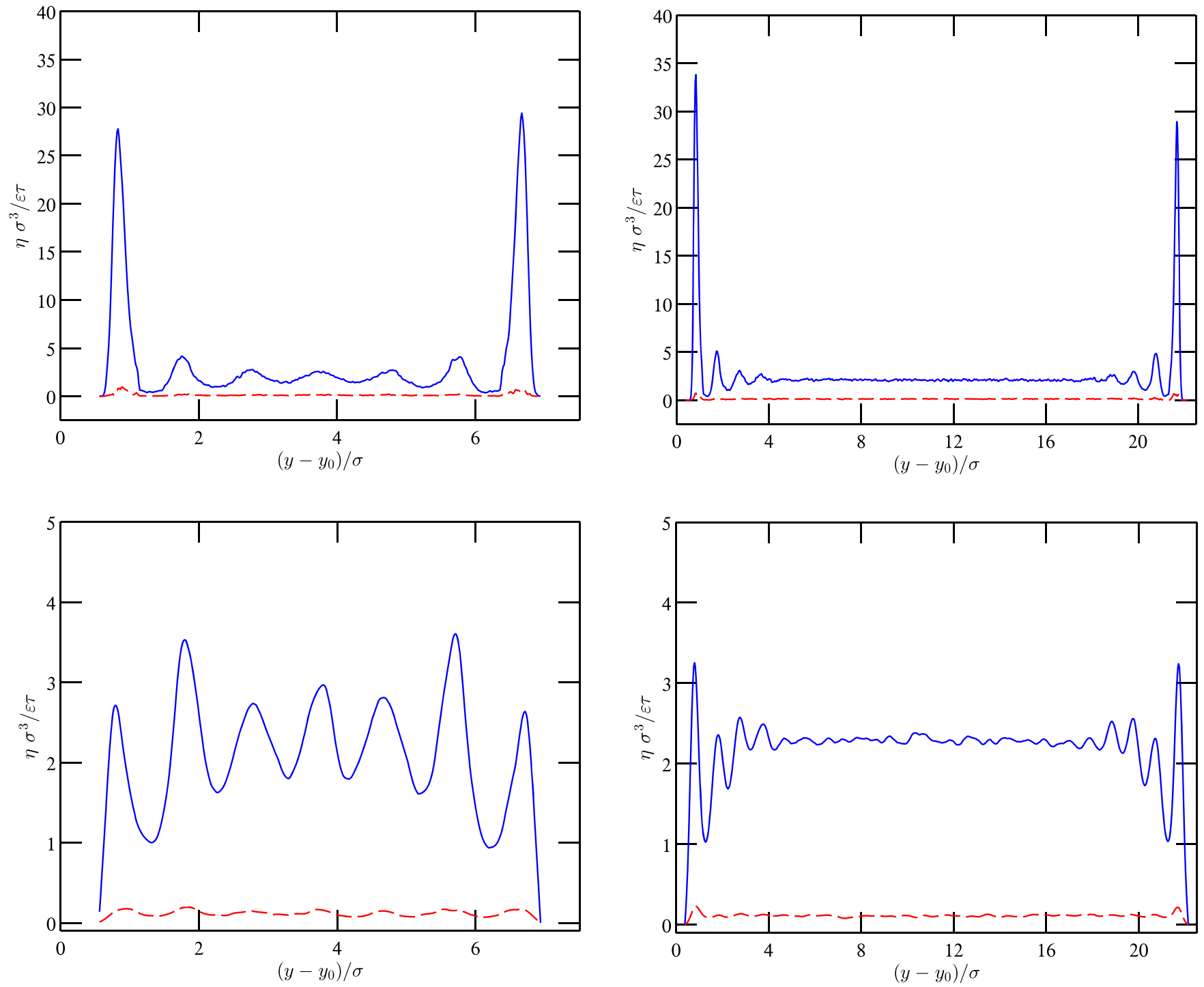

FIG. 5. Kinetic (red dashed line) and configurational (blue solid line) contributions to the viscosity $\left(\eta \sigma^{3} / \varepsilon \tau\right)$ along the channel width and with shear rate $\gamma=0.063 \tau^{-1}$ for hydrophilic $\left(\varepsilon_{w}=\varepsilon\right)$ and hydrophobic $\left(\varepsilon_{w}=0.2 \varepsilon\right)$ substrates are depicted in the top and bottom rows, respectively. The left and right columns show results for channel widths $h=6.43 \sigma$ and $h=21.44 \sigma$, respectively.

(a)

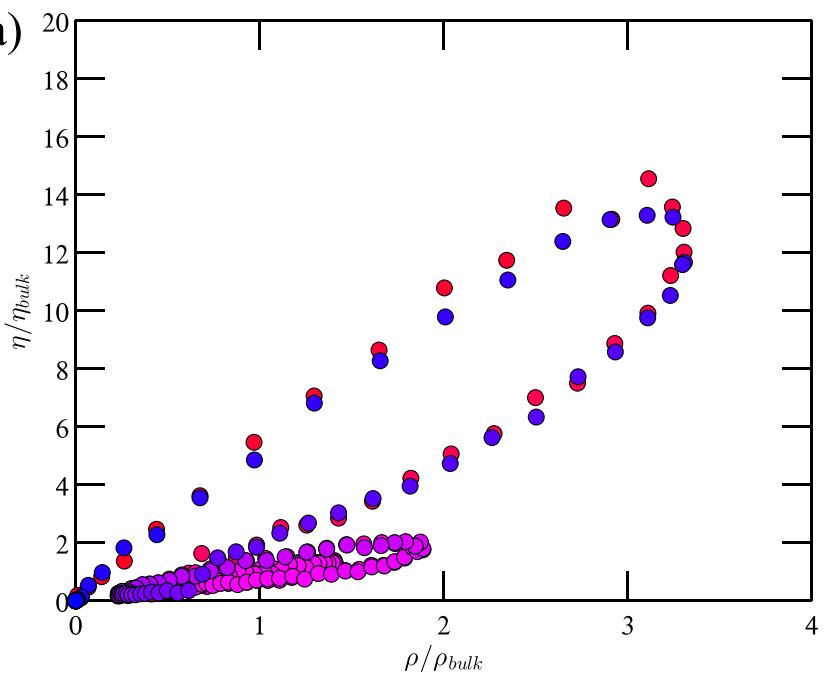

(b)

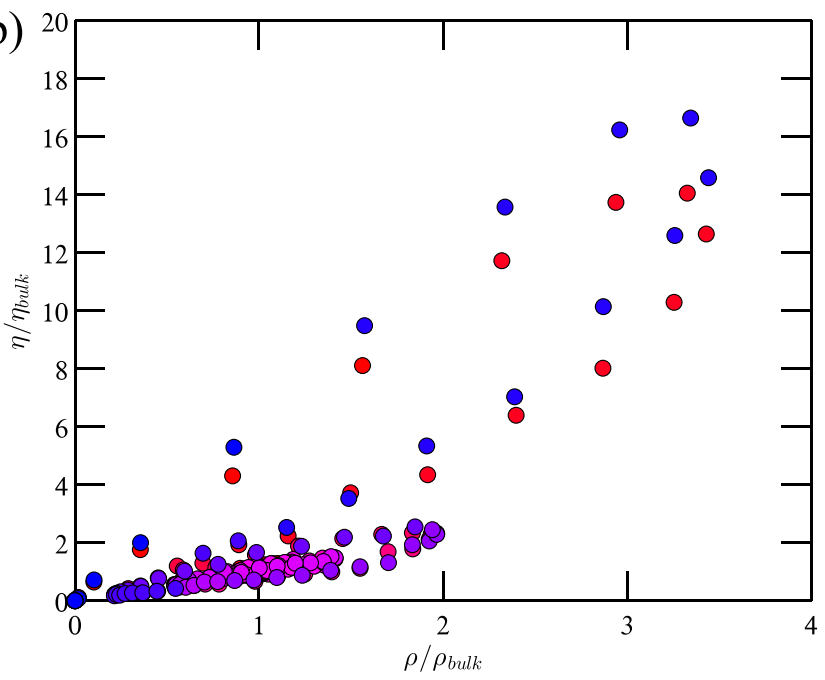

FIG. 6. Normalised density $\left(\rho / \rho_{\text {bulk }}\right)$ vs normalised viscosity $\left(\eta / \eta_{b u l k}\right)$ across the channel for hydrophilic substrates $\left(\varepsilon_{w}=\varepsilon\right)$ and for channel widths $h=6.43 \sigma$ and $21.44 \sigma$ shown in subplots (a) and (b), respectively. The colours are scaled between the static bottom wall (blue) and the sheared top wall (red). As a result, the middle of the channel is represented by purple symbols. The bulk values employed for normalisation are $\rho_{b u l k}=0.81 \sigma^{-3}$ and $\eta_{b u l k}=2.15 \varepsilon \tau / \sigma^{3}$. 
regions, the slopes of the $\partial \eta / \partial \rho$ curve are generally greater than the ones obtained in the middle of the channel. Also, we observe strong non-linear "fan-shaped" structures. These may arise from the bending of the velocity profile in the proximity of the walls, as well as the interactions at the solid-liquid interface, which are ultimately creating the oscillatory structures in both viscosity and density.

The rich behaviour depicted in Fig. 6 exemplifies the inadequacy of using a constant viscosity in mean-field DDFT or coarse-grained models where walls are present. It may also be tempting to dismiss a simple linear relationship for $\eta(\rho)$ for this scenario. However, let us first recognise that for non-repulsive walls, in the layer closest to the wall, the position of the fluid particles is highly organised, leading to a highly peaked density distribution (see Fig. 2). It is thus reasonable to exclude the first layer from our considerations, and therefore only attempt to capture the viscosity behaviour away from this first layer. We thus investigate the data from Fig. 6 by first averaging the data from the top and the bottom walls, therefore exploiting the spatial symmetry in the $y$ direction, and then removing the layer of particles closest to the walls, as shown in Fig. 7 ("MD data" curves).

We see in Fig. 7 a good fit to the data for all but the first molecular layer nearest the walls is given by

$$
\eta=\left(\frac{\eta_{\text {bulk }}}{\rho_{\text {bulk }}}\right) \rho
$$

represented by the black dashed-dotted line. This fit is achieved with an $R^{2}$ value of 0.83 . This law is particularly favourable for implementation in coarse-grained models as it requires knowledge of only the bulk values of density and viscosity and is independent of the degree of confinement of either. Allowing for a confinement-dependent fit and offset values at zero density only improved the fitting quality marginally to a $R^{2}$-value of 0.85 . This means that at this level of description,

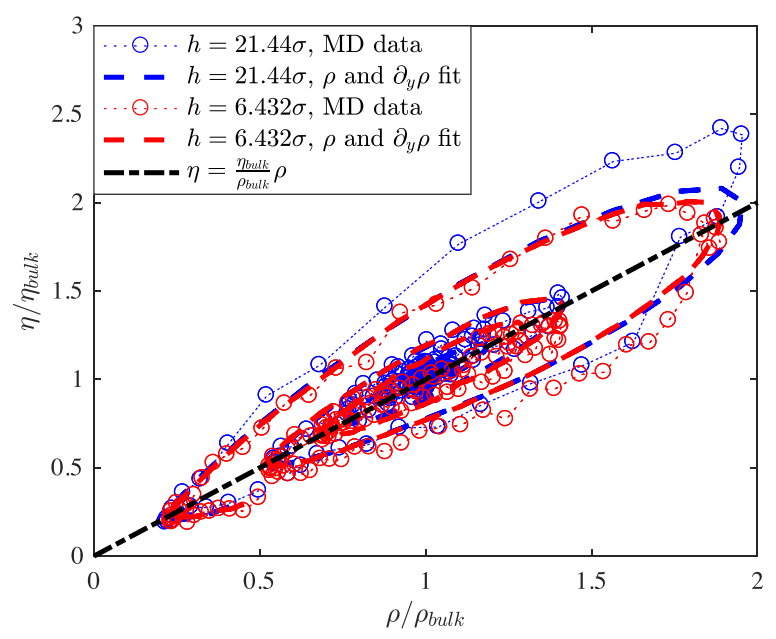

FIG. 7. Viscosity vs. density for two channel widths for hydrophilic substrates and comparison with linear relationship $\eta=\left(\eta_{\text {bulk }} / \rho_{\text {bulk }}\right) \rho$ for all but the first particle layers nearest the walls. The data have been averaged for each channel width exploiting the spatial symmetry in the $y$ direction. The linear relationship requires knowledge of only the bulk values of density and viscosity, and achieves an $R^{2}=0.83$ fit to the data. Futhermore, the thicker dashed lines are plotted using the generalized fit (11), which also includes the density gradients normal to the walls and achieves an $R^{2}=0.95$ fit to the data. the universal law (10) which does not depend on the degree of confinement is justified.

It is noteworthy, however, that the deviations between our MD results and (10) are up to $\pm 40 \%$ for the second layer of fluid from the wall and then improve quickly thereafter. One possibility to improve the quality of the fit would be to include gradients of the density in the direction normal to the wall in the constitutive relationship between density and viscosity. This accounts for the oscillatory behaviour of the deviations in Fig. 7 but would be dependent on the particular wall and fluid interactions being modelled, in contrast to (10).

Thus far in this section, we have argued that a simple linear relationship between density and viscosity can be justified for confined fluids. In particular, this has been done in a way which would be straightforward to implement in coarse-grained models (i.e., with no further MD runs required for different setups) and involves an approximation error not unreasonable given other errors in coarse-graining. However, we also realise that such a simple linear relationship is incapable of retaining any direct knowledge of the fluid layering/structure.

We thus also include gradients of the density in the direction normal to the wall in the constitutive relationship between density and viscosity. The simplest law extending (10) balances the smallest number of fitting parameters with reasonable additional accuracy. We find a generalised law as

$$
\eta=\left(\frac{\eta_{\text {bulk }}}{\rho_{\text {bulk }}}\right)\left(\rho+0.056 \sigma \frac{\partial \rho}{\partial y}\right),
$$

which gives a fit with an $R^{2}$ value of 0.95 for all of the data as used in the linear law above, and plotted in Fig. 7.

The fits are displayed in Fig. 7 with blue and red dashed lines corresponding to their respective MD data points. Figure 8 also plots the fit of (11) to the MD data by explicitly showing the strong dependence on $\rho$ and the weaker dependence on $\partial_{y} \rho$ to account for the oscillations in the fluid structure near to the walls. We reiterate, however, that this generalized law is not as immediately useful for coarse-grained

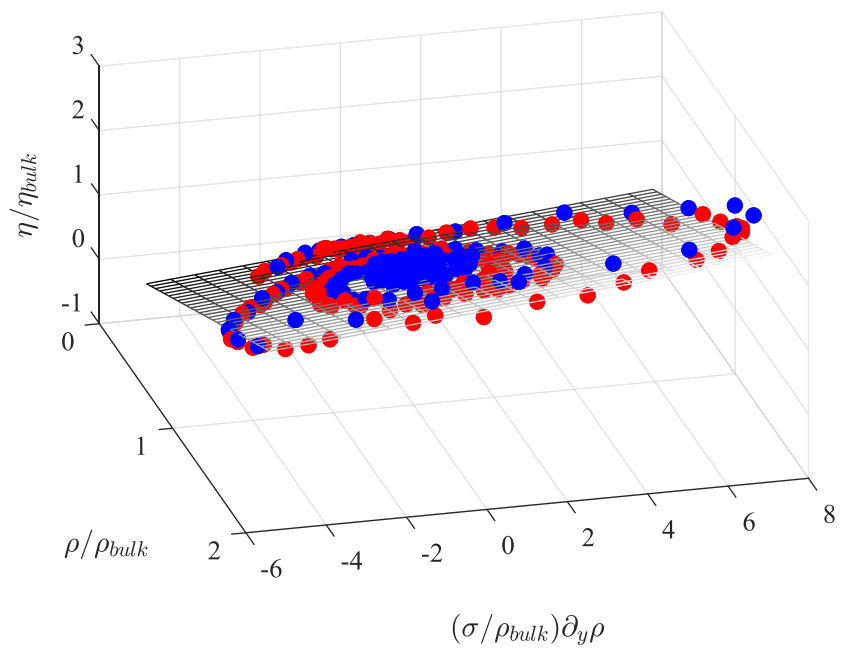

FIG. 8. Normalised viscosity $\left(\eta / \eta_{\text {bulk }}\right)$ plotted depending on normalised density $\left(\rho / \rho_{\text {bulk }}\right)$ and normalised density gradients $\left(\sigma / \rho_{\text {bulk }}\right) \partial_{y} \rho$. The plane shows the fit described in (11). The two confinement situations are coloured as in Fig. 7. 
models as the coefficient in front of the gradient term may depend on the oscillatory structure of the fluid density, which in turn depends on the particular fluid and solid interactions being modelled. The LJ system studied here has been chosen as an extreme prototype, with significant structure and hence large density gradients. Then for other fluids, the additional term in (11) would have an even smaller effect and thus leading to an even stronger recommendation of the linear law in (10).

Given that for the above viscosity-density relationships, we have excluded the first layer of fluid atoms, in Appendix C, we also discuss the possibility to include the first layer of fluid particles by fitting the values at the peaks of the fluid density to an exponential law. This idea needs, however, to be tested for a wider range of parameters.

\section{DISCUSSION AND CONCLUSIONS}

Detailed MD simulations have been performed to scrutinise the effect of nanoconfinement on the mass transport properties of a LJ fluid under planar Couette flow conditions. Several nanoconfinement conditions were considered by changing the channel width and the hydrophilicity of the walls.

Nanoconfinement leads to fluid layering, which manifests itself in density oscillations in the proximity of the solid-fluid interface, and is observed for all channel widths. Away from the solid surfaces, bulk conditions are recovered, as expected. Density oscillations depend on the forces exerted by the wall atoms. Close to the walls, the components of the stress tensors show a layering that is a consequence of fluid adsorption: the adhesion of fluid particles onto the surface, especially in hydrophilic conditions, creates a layer that is largely immobilised leading to the rich behaviours in densities, stresses, and viscosities. Outside the region of wall influence, the stress tensor becomes isotropic.

Using the velocity and stress-tensor profiles and assuming a Newtonian relation between the off-diagonal stress tensor and the velocity gradient, we computed the local shear viscosity within the nanochannels. We then studied the local relationship between density and viscosity across the channel. By discounting the nearest-wall molecular layer, which exhibits strong non-Newtonian properties, we found that a simple linear relationship [see Eq. (10)] between viscosity and density performs well, even for strongly inhomogeneous fluids and high degrees of confinement. These results support careful use of the simple linear constitutive law between these two quantities in coarse-grained meso- and macroscopic models. We can improve the fit further by employing a generalised constitutive relationship between viscosity and density which includes the gradients of the density in the direction normal to the wall (but this would depend on the particular fluid and solid interactions being modelled).

In conclusion, our results may be used in conjunction with coarse-grained models such as DDFT and NS-like formalisms in Refs. 31 and 32. In nanoscopic flows involving multiple phases, such as nanodroplet spreading, density inhomogeneities are caused by both the presence of wall interactions and fluid-fluid interfaces. Further atomistic studies involving multiple phases and more complex flow profiles thus have to be performed in order to probe the relationship between viscosity and density and to inform coarse-grained models for the corresponding systems. ${ }^{68,69}$

\section{ACKNOWLEDGMENTS}

M.M., M.F., E.C., and P.A. acknowledge financial support from the Italian Ministry of Education, University and Research through the THERMALSKIN ("Revolutionary surface coatings by carbon nanotubes for high heat transfer efficiency"; FIRB 2010, Grant No. RBFR10VZUG) and the NANOBRIDGE projects ("Heat and mass transport in NANOstructures by molecular dynamics, systematic model reduction, and non-equilibrium thermodynamics"; PRIN 2012, Grant No. 2012LHPSJC). M.M., M.F., E.C., and P.A. thank the CINECA (Iscra C project DISCALIN) and the Politecnico di Torino's DAUIN High Performance Computing Initiative for the availability of high performance computing resources and support. M.M. thanks the Chemical Engineering Department of Imperial College (IC) for hospitality and financial support during a visit in 2015 as part of his Masters research at Politecnico di Torino. A.N. acknowledges financial support from IC through a DTG International Studentship, and C.B., P.Y., D.N.S., B.D.G., and S.K. acknowledge financial support from the EPSRC ("Statistical mechanics of soft matter: Derivation, analysis and implementation of dynamic density functional theories"; Grant No. EP/L025159/1 and "Multiscale Analysis of Complex Interfacial Phenomena (MACIPh): Coarse graining, molecular modelling, stochasticity, and experimentation"; Grant No. EP/L020564/1), the ERC ("Complex Interfacial Flows: From the Nano-to the Macroscale"; Advanced Grant Nos. 247031) and EP/K503733: EPSRC-IC Pathways to Impact-Impact Acceleration Award.

\section{APPENDIX A: EFFECTIVENESS OF THE THERMOSTAT}

In Fig. 9, we provide the temperature profiles considering different degrees of fluid confinement and hydrophilicity,
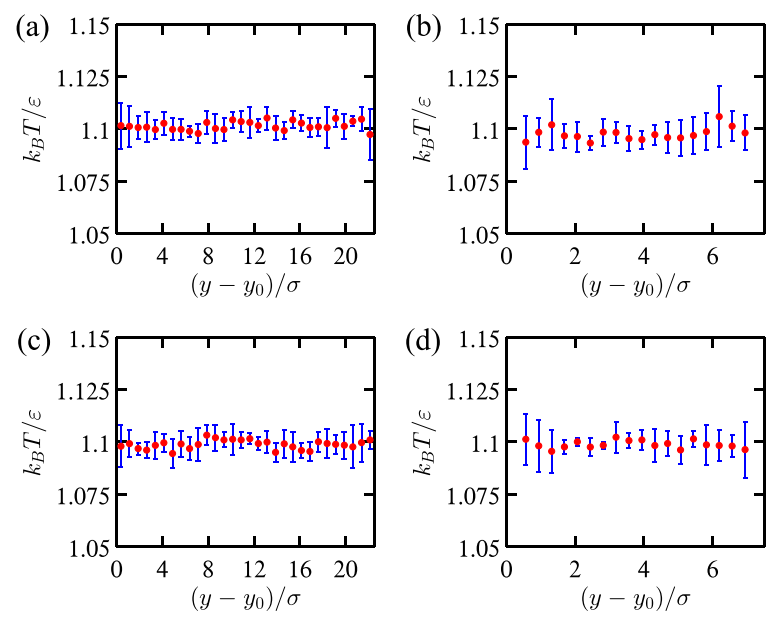

FIG. 9. Temperature profile $\left(k_{B} T / \varepsilon\right)$ of the LJ fluid along the channel width: (a) $h=21.44 \sigma$ and $\varepsilon_{w}=\varepsilon$; (b) $h=6.43 \sigma$ and $\varepsilon_{w}=\varepsilon$; (c) $h=21.44 \sigma$ and $\varepsilon_{w}=0.2 \varepsilon ;(\mathrm{d}) h=6.43 \sigma$ and $\varepsilon_{w}=0.2 \varepsilon$. 
which demonstrate the effectiveness of the adopted Langevin thermostat.

\section{APPENDIX B: SLIP LENGTH}

The fluid velocity at the solid-fluid interface is usually quantified by the slip length $L_{s}$, which is defined through ${ }^{18,23,39}$

$$
v_{s}=\left.L_{s} \frac{\partial v}{\partial y}\right|_{h / 2} .
$$

Here, $v_{s}$ is the tangential velocity at the solid surface and $\partial v / \partial y$ is the velocity gradient normal to the wall. Here, due to the strong anisotropy in the first few fluid layers close to the wall, we use velocity gradients close to the center of the domain, where bulk properties of the fluid are recovered. The main factors affecting slip are the energy of wall-fluid interactions and the shear rate..$^{2,23}$ It should be noted that the exact value of the slip length can have a profound impact even on macroscopic flows, e.g., at the moving contact line (see, e.g., the work of Sibley et al. ${ }^{70}$ ).

In Fig. 10, we present slip length measurements for hydrophilic and hydrophobic surfaces as a function of the shear rate. In the case of hydrophilic walls, $L_{s}$ is almost constant and negative of the order of $-\sigma$, meaning that the effective no-slip boundary plane is located in the fluid domain.

For hydrophobic walls, $L_{s}$ is positive. Figure 11 depicts the slip length $L_{s}$ as a function of the shear rate measured within the fluid $\gamma_{m}$, showing a divergent behaviour as $\gamma_{m} \rightarrow \gamma_{c}$. In other words, the slip length is almost constant at relatively low shear rates, whereas it increases non-linearly close to a critical shear rate $\gamma_{c}$. The results for $L_{s}$ vs. $\gamma$ are then fitted to

$$
\frac{L_{s}}{L_{s 0}}=\left(1-\frac{\gamma}{\gamma_{c}}\right)^{-\alpha_{c}} \text {. }
$$

In Fig. 11, it is shown that this leads to a good fit for $\alpha_{c}=0.5$, in good agreement with previous results in the literature. ${ }^{18}$ We note, however, that including $\alpha_{c}$ as a fitting parameter leads to a better quality fit with $\alpha_{c}=2.52$, suggesting a modified asymptotic behaviour. Further computations for higher

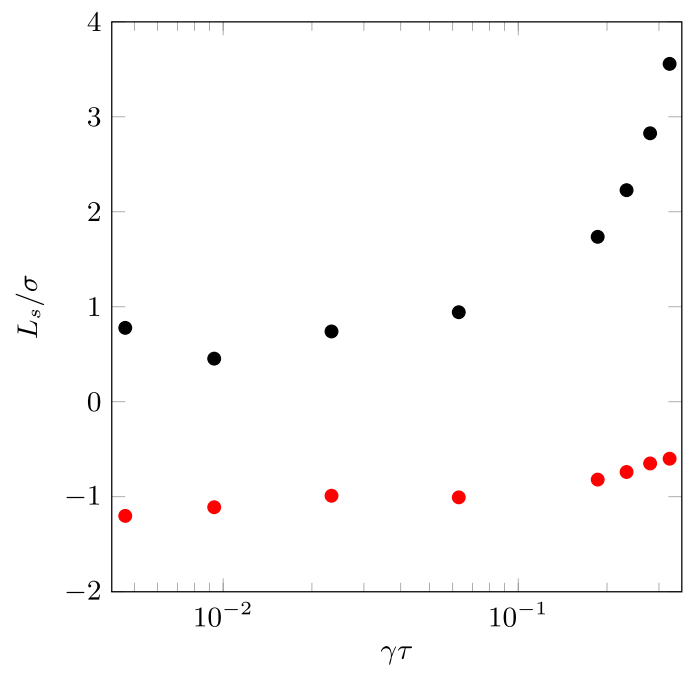

FIG. 10. Slip length $\left(L_{s} / \sigma\right)$ as a function of the shear rate $(\gamma \tau)$. The nanochannel width is $h=21.44 \sigma$; black circles and red circles correspond to $\varepsilon_{w}=0.2 \varepsilon$ and $\varepsilon_{w}=\varepsilon$, respectively.

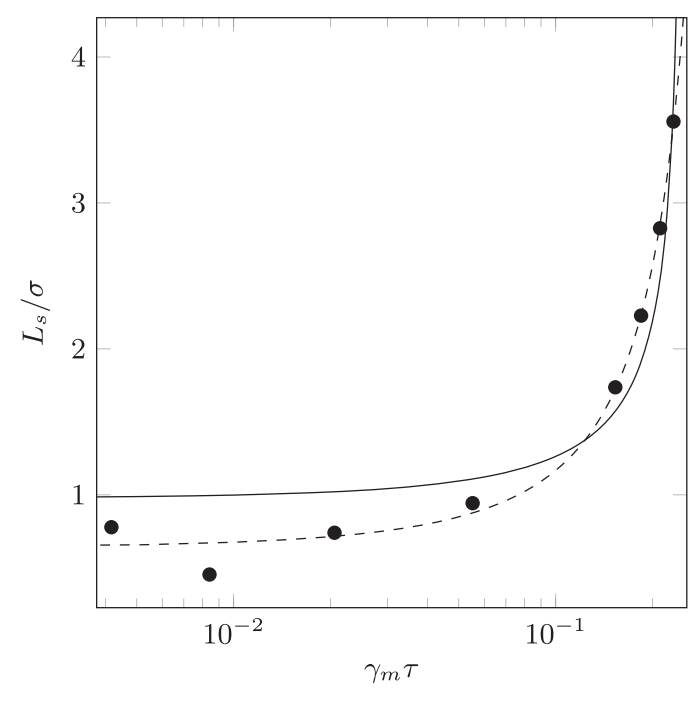

FIG. 11. Slip length $L_{s}$ as a function of the effective shear rate $\gamma_{m}$ as measured in the fluid for $h=21.44 \sigma$ and $\varepsilon_{w}=0.2 \varepsilon_{f}$. The solid line is a fit to Eq. (B2) for $\alpha=0.5$, according to the results by Thompson and Troian, ${ }^{18}$ leading to $\gamma_{c}=0.25 \tau^{-1}$ and $L_{s 0}=0.98 \sigma$. The dashed line depicts a fit if $\alpha_{c}$ is included as a fitting parameter, resulting in $\alpha_{c}=2.52, \gamma_{c}=0.47 \tau^{-1}$, and $L_{s 0}=0.64 \sigma$.

slip lengths are needed to determine the asymptotic behaviour conclusively.

\section{APPENDIX C: DENSITY AND VISCOSITY: DENSITY PEAKS}

In Sec. III E, we argued that the first layer of fluid particles close to the wall needs to be considered separately. Here, we attempt to extend our theory to the first layer, by fitting data at the density peaks along the channel (without including any dependency on the density gradient). In particular, we fit the normalised values of the viscosity peaks $\left(\eta / \eta_{\text {bulk }}\right)$ as a function of the normalised values of the density peaks $\left(\rho / \rho_{\text {bulk }}\right)$. This will allow us some coarse measure of how viscosity and density are related up to the walls.

Specifically, we observe that moving from the solid-liquid interface towards the bulk region, the peak values of the

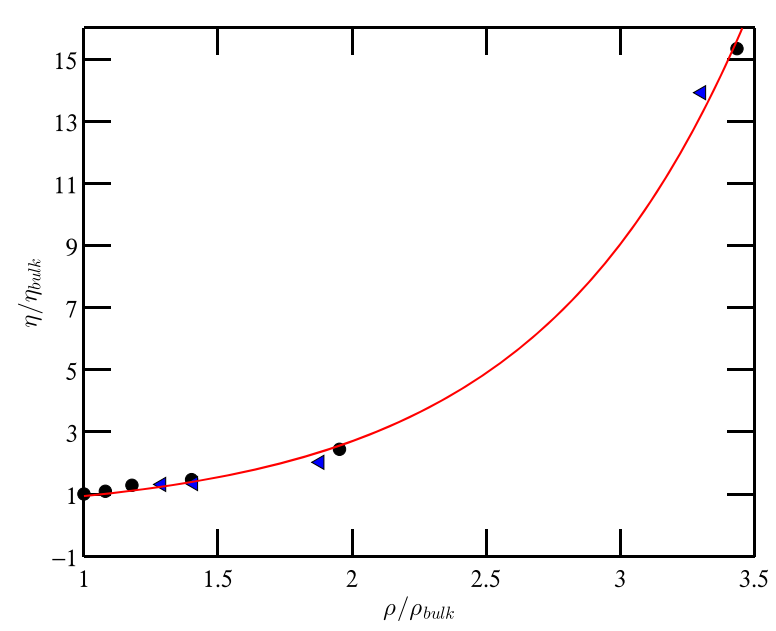

FIG. 12 . Normalised density $\left(\rho / \rho_{\text {bulk }}\right)$ vs. normalised viscosity $\left(\eta / \eta_{\text {bulk }}\right)$ at the density peaks for channel widths $h=21.44 \sigma$ (black circles) and $h$ $=6.43 \sigma$ (blue triangles). The red solid line is a fit to Eq. (C1), with fitting parameters $a=0.19, b=1.28$, and $c=0.31\left(R^{2}=0.9974\right)$. 
density and viscosity profiles decrease exponentially (see Figs. 2 and 5). Inspired by this decay, we postulate the following law:

$$
\frac{\eta}{\eta_{\text {bulk }}}=a \exp \left(b \frac{\rho}{\rho_{\text {bulk }}}\right)+c .
$$

In Fig. 12, our data are fitted to this law, showing a good agreement for different degrees of confinement. However, we reiterate that this is the fit only to the peak values, and as such is a speculative attempt to propose a fitting law for the entire channel up to the walls. In most applications using meso- or macroscopic models, we recommend (10) (or (11) if hoping to also capture some of the behaviour due to the fluid oscillations near the walls) in the first instance even if it cannot account for the nearest-wall molecular layer.

${ }^{1}$ G. E. Totten, Handbook of Hydraulic Fluid Technology (CRC Press, 2011). ${ }^{2}$ J. Judy, D. Maynes, and B. W. Webb, Int. J. Heat Mass Transfer 45(17), 3477-3489 (2002).

${ }^{3}$ D. D. Braun and M. R. Rosen, Rheology Modifiers Handbook: Practical Use and Application (Cambridge University Press, 2008).

${ }^{4}$ T. Geninatti, R. L. Hood, G. Bruno, P. Jain, E. Nicolov, A. Ziemys, and A. Grattoni, Materials 8(8), 5276-5288 (2015).

${ }^{5}$ A. Gizzatov, J. Key, S. Aryal, J. Ananta, A. Cervadoro, A. L. Palange, M. Fasano, C. Stigliano, M. Zhong, D. Di Mascolo et al., Adv. Funct. Mater. 24(29), 4584-4594 (2014).

${ }^{6}$ D. L. Klass and T. W. Martinek, J. Appl. Phys. 38(1), 67-74 (1967).

${ }^{7}$ E. Chiavazzo, M. Fasano, P. Asinari, and P. Decuzzi, Nat. Commun. 5, 3565 (2014).

${ }^{8}$ M. Fasano, E. Chiavazzo, and P. Asinari, Nanoscale Res. Lett. 9(1), 559 (2014).

${ }^{9}$ P. Yatsyshin, N. Savva, and S. Kalliadasis, Phys. Rev. E 87, 020402(R) (2013).

${ }^{10}$ A. Nold, D. N. Sibley, B. D. Goddard, and S. Kalliadasis, Phys. Fluids 26, 072001 (2014).

${ }^{11}$ D. N. Sibley, A. Nold, N. Savva, and S. Kalliadasis, Phys. Fluids 25(9), 092111 (2013).

${ }^{12}$ P. M. J. Trevelyan, S. Kalliadasis, J. H. Merkin, and S. K. Scott, J. Eng. Math. 42(1), 45-64 (2002).

${ }^{13}$ M. Fasano, D. Borri, E. Chiavazzo, and P. Asinari, Appl. Therm. Eng. 101, 762 (2016).

${ }^{14}$ C.-L. Navier, Mem. Acad. Sci. Inst. Fr. 6, 389-440 (1823).

${ }^{15}$ N. Asproulis and D. Drikakis, Phys. Rev. E 81(6), 061503 (2010).

${ }^{16}$ S.-C. Yang, Microfluid. Nanofluid. 2(6), 501-511 (2006).

${ }^{17}$ H. Zhang, Z. Zhang, and H. Ye, Microfluid. Nanofluid. 12(1-4), 107-115 (2012).

${ }^{18}$ P. A. Thompson and S. M. Troian, Nature 389(6649), 360-362 (1997).

${ }^{19}$ E. Lauga, M. Brenner, and H. Stone, "Microfluidics: The no-slip boundary condition," in Springer Handbook of Experimental Fluid Mechanics (Springer, 2007), pp. 1219-1240.

${ }^{20}$ K. P. Travis, B. D. Todd, and D. J. Evans, Phys. Rev. E 55(4), 4288-4295 (1997).

${ }^{21}$ P. A. Thompson and M. O. Robbins, Phys. Rev. A 41(12), 6830 (1990).

${ }^{22}$ N. V. Priezjev, Phys. Rev. E 80(3), 031608 (2009).

${ }^{23}$ N. V. Priezjev, Phys. Rev. E 75(5), 051605 (2007).

${ }^{24}$ R. L. Rowley and M. M. Painter, Int. J. Thermophys. 18(5), 1109-1121 (1997).

${ }^{25}$ K. Meier, A. Laesecke, and S. Kabelac, J. Chem. Phys. 121(8), 3671-3687 (2004).

${ }^{26}$ G. Galliéro, C. Boned, and A. Baylaucq, Ind. Eng. Chem. Res. 44(17), 6963-6972 (2005).

${ }^{27}$ R. Hartkamp and S. Luding, "Anisotropic Lennard-Jones fluids in a nanochannel," in 7th International Conference on Multiphase Flow, ICMF 2010.

${ }^{28}$ R. Hartkamp, A. Ghosh, T. Weinhart, and S. Luding, J. Chem. Phys. 137(4), 044711 (2012).
${ }^{29}$ R. Hartkamp, B. D. Todd, and S. Luding, J. Chem. Phys. 138(24), 244508 (2013).

${ }^{30}$ A. Nold, D. N. Sibley, B. D. Goddard, and S. Kalliadasis, Math. Modell. Nat. Phenom. 10(4), 111-125 (2015).

${ }^{31}$ B. D. Goddard, A. Nold, N. Savva, G. A. Pavliotis, and S. Kalliadasis, Phys. Rev. Lett. 109, 120603 (2012).

${ }^{32}$ B. D. Goddard, A. Nold, N. Savva, P. Yatsyshin, and S. Kalliadasis, J. Phys.: Condens. Matter 25(3), 035101 (2013).

${ }^{33}$ J.-P. Hansen and I. R. McDonald, Theory of Simple Liquids: With Applications to Soft Matter (Academic Press, 2013).

${ }^{34}$ M. P. Allen, Computational Soft Matter: From Synthetic Polymers to Proteins (John von Neumann Institute for Computing, 2004), Vol. 23, pp. $1-28$.

${ }^{35}$ S. Plimpton, J. Comput. Phys. 117, 1-19 (1995).

${ }^{36}$ X. Yong and L. T. Zhang, J. Chem. Phys. 138(8), 084503 (2013).

${ }^{37}$ A. Ghosh, R. Paredes, and S. Luding, "Poiseuille flow in a nanochanneluse of different thermostats," in PARTEC 2007, Congress on Particle Technology, edited by W. Peukert and C. Schreglmann (University of Erlangen-Nuremberg, Institute of Particle Technology, 2008).

${ }^{38}$ J. J. Thalakkottor and K. Mohseni, Phys. Rev. E 94(2), 023113 (2016).

${ }^{39}$ N. V. Priezjev, Microfluid. Nanofluid. 14(1-2), 225-233 (2013).

${ }^{40}$ N. V. Priezjev and S. M. Troian, J. Fluid Mech. 554, 25-46 (2006).

${ }^{41}$ H. J. C. Berendsen, J. P. M. Postma, W. F. van Gunsteren, A. Dinola, and J. R. Haak, J. Chem. Phys. 81(8), 3684-3690 (1984).

${ }^{42}$ T. Schneider and E. Stoll, Phys. Rev. B 17(3), 1302 (1978).

${ }^{43}$ S. Bernardi, B. Todd, and D. J. Searles, J. Chem. Phys. 132(24), 244706 (2010)

${ }^{44}$ I. Bitsanis, J. J. Magda, M. Tirrell, and H. T. Davis, J. Chem. Phys. 87(3), 1733-1750 (1987).

${ }^{45}$ M. Tsige and G. S. Grest, J. Chem. Phys. 120(6), 2989-2995 (2004).

${ }^{46}$ D. J. Evans and G. P. Morriss, Phys. Rev. A 30, 1528-1530 (1984).

${ }^{47}$ A. Niavarani and N. V. Priezjev, Phys. Rev. E 81(1), 011606 (2010).

${ }^{48}$ T. Soddemann, B. Dünweg, and K. Kremer, Phys. Rev. E 68(4), 046702 (2003).

${ }^{49}$ N. V. Priezjev, A. A. Darhuber, and S. M. Troian, Phys. Rev. E 71(4), 041608 (2005).

${ }^{50}$ S. T. O'Connell and P. A. Thompson, Phys. Rev. E 52(6), R5792 (1995).

${ }^{51}$ F. Wang and Y. Zhao, Acta Mech. Solida Sin. 24(2), 101-116 (2011).

${ }^{52}$ J. N. Israelachvili, Intermolecular and Surface Forces, 3rd ed. (Academic Press, 2011).

${ }^{53}$ K. P. Travis and K. E. Gubbins, J. Chem. Phys. 112(4), 1984-1994 (2000).

${ }^{54}$ R. Kjellander and S. Sarman, Mol. Phys. 70(2), 215-237 (1990).

${ }^{55}$ R. Kjellander and S. Sarman, Mol. Phys. 74(3), 665-688 (1991).

${ }^{56}$ L. D. Gelb and R. M. Lynden-Bell, Phys. Rev. B 49(3), 2058 (1994).

${ }^{57}$ S. Dietrich, in Phase Transitions and Critical Phenomena, Vol. 12, edited by C. Domb and J. Lebowitz (Academic Press, London, 1988).

${ }^{58}$ R. Devi, S. Srivastava, and K. Tankeshwar, J. Chem. Phys. 143(2), 024506 (2015).

${ }^{59}$ R. Evans and U. M. B. Marconi, J. Chem. Phys. 86(12), 7138-7148 (1987).

${ }^{60}$ R. Evans and A. O. Parry, J. Phys.: Condens. Matter 2(S), SA15 (1990).

${ }^{61}$ P. Yatsyshin, N. Savva, and S. Kalliadasis, J. Chem. Phys. 142(3), 034708 (2015).

${ }^{62}$ P. Yatsyshin, N. Savva, and S. Kalliadasis, J. Phys.: Condens. Matter 27, 275104 (2015).

${ }^{63}$ P. Yatsyshin, A. O. Parry, and S. Kalliadasis, J. Phys.: Condens. Matter 28(27), 275001 (2016).

${ }^{64}$ J. H. Irving and J. G. Kirkwood, J. Chem. Phys. 18(6), 817-829 (1950).

${ }^{65}$ M. Barisik and A. Beskok, Microfluid. Nanofluid. 11(3), 269-282 (2011).

${ }^{66}$ B. D. Todd, D. J. Evans, and P. J. Daivis, Phys. Rev. E 52(2), 1627 (1995).

${ }^{67}$ D. Rajagopalan, R. C. Armstrong, and R. A. Brown, J. Non-Newtonian Fluid Mech. 36, 159-192 (1990).

${ }^{68}$ B. D. Goddard, A. Nold, and S. Kalliadasis, J. Chem. Phys. 138, 144904 (2013).

${ }^{69}$ B. D. Goddard, A. Nold, and S. Kalliadasis, J. Chem. Phys. 145, 214106 (2016).

${ }^{70}$ D. N. Sibley, A. Nold, N. Savva, and S. Kalliadasis, J. Eng. Math. 94, 19-41 (2015). 